\title{
Personality traits in rats predict vulnerability and resilience to developing stress-induced depression- like behaviors, HPA axis hyper-reactivity and brain changes in PERK1/2 activity
}

\author{
Jorge E. Castro, Shanaz Diessler, Emilio Varea, Cristina Márquez, \\ Marianne H. Larsen, M. Isabel Cordero, Carmen Sandi *
}

Laboratory of Behavioral Genetics, Brain Mind Institute, School of Life Sciences, École Polytechnique Fédérale de Lausanne (EPFL), Lausanne, Switzerland

Received 30 August 2011; received in revised form 14 December 2011; accepted 14 December 2011

\section{KEYWORDS \\ Stress; \\ Personality; \\ Depression; \\ Anxiety; \\ Exploration; \\ Corticosterone; \\ ERK1/2}

\begin{abstract}
Summary Emerging evidence indicates that certain behavioral traits, such as anxiety, are associated with the development of depression-like behaviors after exposure to chronic stress. However, single traits do not explain the wide variability in vulnerability to stress observed in outbred populations. We hypothesized that a combination of behavioral traits might provide a better characterization of an individual's vulnerability to prolonged stress. Here, we sought to determine whether the characterization of relevant behavioral traits in rats could aid in identifying individuals with different vulnerabilities to developing stress-induced depressionlike behavioral alterations. We also investigated whether behavioral traits would be related to the development of alterations in the hypothalamic-pituitary-adrenal axis and in brain activity as measured through phosphorylation of extracellular signal-regulated kinase $1 / 2(E R K 1 / 2)-$ in response to an acute stressor following either sub-chronic ( 2 weeks) or chronic (4 weeks) unpredictable stress (CUS). Sprague-Dawley rats were characterized using a battery of behavioral tasks, and three principal traits were identified: anxiety, exploration and activity. When combined, the first two traits were found to explain the variability in the stress responses. Our findings confirm the increased risk of animals with high anxiety developing certain depression-like behaviors (e.g., increased floating time in the forced swim test) when progressively exposed to stress. In contrast, the behavioral profile based on combined low anxiety and low exploration was resistant to alterations related to social behaviors, while the high anxiety and low exploration profile displayed a particularly vulnerable pattern of physiological and neurobiological responses after sub-chronic stress exposure. Our findings indicate important differences in
\end{abstract}

\footnotetext{
* Corresponding author at: Laboratory of Behavioral Genetics, Brain Mind Institute, École Polytechnique Fédérale de Lausanne (EPFL), Station 19, CH-1015 Lausanne, Switzerland. Tel.: +41 021693 1762; fax: +41 0216939636.

E-mail address: carmen.sandi@epfl.ch (C. Sandi).
} 
animals' vulnerability and/or resilience to the effects of repeated stress, particularly during initial or intermediate levels of stress exposure, and they highlight that the behavioral inhibition profile of an animal provides a particular susceptibility to responding in a deleterious manner to stress.

(C) 2011 Elsevier Ltd. All rights reserved.

\section{Introduction}

Stress can influence the development and exacerbate the symptoms of a variety of psychiatric disorders, including depression, anxiety, posttraumatic stress disorder and schizophrenia (McEwen, 2004; de Kloet et al., 2005, 2007; Sandi and Richter-Levin, 2009). An increasing number of animal models based on stress interventions have been shown to effectively mimic a variety of psychopathological alterations (Willner, 2005; Renthal et al., 2007; Stam, 2007; Ilin and Richter-Levin, 2009). In both animals and humans, excessive and/or enduring stress has been found to cause structural and neurochemical alterations in several brain structures, especially in the hippocampus (Lupien et al., 1998; Sheline et al., 1999; McEwen, 2000; Pham et al., 2003; Bisaz et al., 2011), the prefrontal cortex (Drevets et al., 1997; Rajkowska, 2000; Holmes and Wellman, 2009) and the amygdala (Sandi et al., 2008; Mitra et al., 2009; Roozendaal et al., 2009).

The importance of individual differences in the deleterious effects of stress is gaining recognition. Some individuals show a high vulnerability to stress, whereas others are resilient to developing stress-induced psychopathological alterations (Drugan et al., 1989; Rudolph and Hammen, 1999; Oitzl et al., 2000; New et al., 2009; Sandi and Richter-Levin, 2009; Oitzl et al., 2010; Stiller et al., 2011). Understanding the sources of such differences in vulnerability to stress is a major challenge for contemporary research. A current view suggests that individual differences are the result of the interactions among genetic factors (de Rijk and de Kloet, 2005), predisposing early life experiences and major life stressors (McEwen, 2003). However, the specific underlying mechanisms that determine an individual's vulnerability to stress are not well understood.

Identifying behavioral and/or physiological factors (e.g., personality traits and hormonal responses to stress) capable of predicting an individual's psychopathological vulnerability or resistance to the deleterious effects of stress would help to identify the underlying neurobiological factors. Individual differences in coping style and personality traits have been shown to be associated with resilience to stress or, conversely, with stress-induced depression. One of the most studied factors is the neuroticism-anxiety trait, which is recognized as an important risk factor for the development of depression (Ball and Schottenfeld, 1997; Wang et al., 2002; Sandi and RichterLevin, 2009). The biological significance of neuroticism-anxiety as a predictor of stress-related disorders is supported by a link between this personality trait and basal cortisol levels (Adler et al., 1997; Lindfors and Lundberg, 2002). Using the chronic unpredictable stress (CUS) model of depression (Willner, 1997, 2005) in rats, we have identified an association between anxiety trait and both increased vulnerability to stress-induced depression-like symptoms and the response of the amygdala to emotional cues (Sandi et al., 2008).

Despite the identified role of anxiety trait in the link between stress and depression, only a reduced percentage of the variance of depressive symptomatology are typically explained by anxiety trait (Sandi and Richter-Levin, 2009). This suggests that single personality traits are not to fully explain the variability observed in vulnerability to stress (Hennig, 2004) and that a combination of behavioral traits might better predict an individual's vulnerability to develop depression-like alterations following chronic stress. In fact, other personality traits, such as impulsive sensation seeking, aggression, activity or sociability, have also been shown to be associated with an individual's susceptibility to depression (Ball, 1995; Wang et al., 2004). Cortisol studies have further confirmed the biological significance of these traits (Ballenger et al., 1983; Mazur, 1995; Gunnar et al., 1997; Rosenblitt et al., 2001) and there have been a few studies that successfully linked some of these traits - e.g., impulsive sensation seeking - with hippocampal function (Pickering, 2004).

Here, we aimed to determine whether a combination of behavioral, personality-like traits in rats would help to identify behavioral profiles linked to the development of symptom-specific alterations in the response to stress. To evaluate the differential vulnerability, different groups of rats previously characterized based on their personality-like traits - underwent either 2 or 4 weeks of CUS, and their subsequent behavior in a battery of depression-related tests and their glucocorticoid responses were examined. In addition, we evaluated the expression levels of the mitogenactivated protein kinase extracellular signal-regulated kinase $1 / 2($ ERK1/2) in different brain regions. We focused on ERK1/2 because it plays an important role as an effector molecule in the actions of CRF in different brain regions, including the hippocampus and the amygdala (Refojo et al., 2005; Silberstein et al., 2009) and, in addition, because it has been suggested to play a role in stress, memory, plasticity and depression (Mazzucchelli and Brambilla, 2000; Shen et al., 2004; Qi et al., 2006; Reul and Chandramohan, 2007; Silberstein et al., 2009).

\section{Materials and methods}

\subsection{Animals}

A total of 156 adult male Sprague-Dawley rats (Charles River Laboratories, France) were used (approximately three months old and body weight of 250-275 g at the beginning of the experiment). The rats were housed two per cage in standard plastic cages ( $1177 \mathrm{~cm}^{2}$ surface area) and maintained with ad libitum access to food under controlled light ( $12 \mathrm{~h}$ light/dark cycle; lights on at $0700 \mathrm{~h})$ and temperature $\left(22 \pm 2{ }^{\circ} \mathrm{C}\right)$ conditions. The experimental procedures were approved by the Cantonal Veterinary Authorities (Vaud, Switzerland).

\subsection{General experimental procedures}

The rats were allowed to acclimatize to the housing conditions for 7 days before any experimental procedures were 


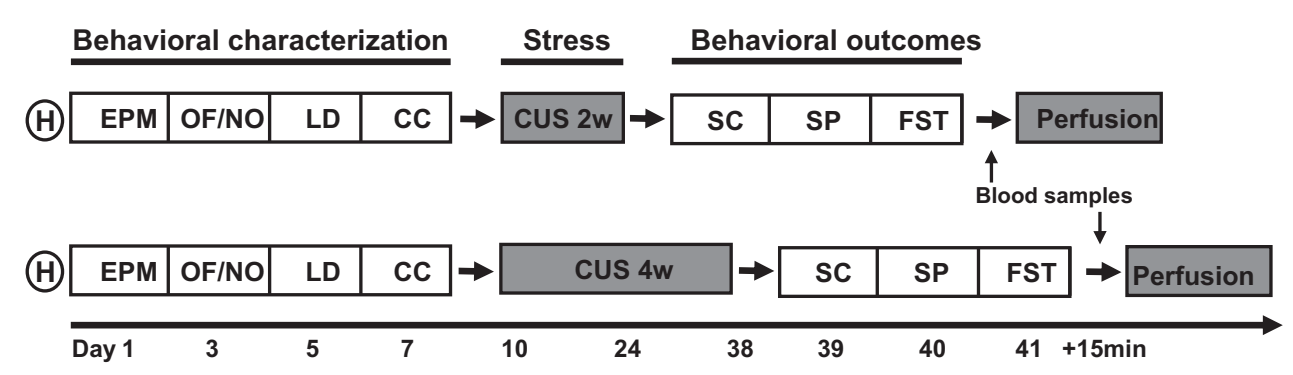

Figure 1 Schematic plan of the experimental procedures. After handling $(\mathrm{H})$, the rats were characterized using the elevated plus maze (EPM), the open field and novel object test (OF/NO), the light and dark box (LD) and the circular corridor (CC). After three days of rest, the rats were submitted to the chronic unpredictable stress protocol (CUS) for either 2 or 4 weeks. Depression-like behaviors were evaluated using the saccharin consumption test (SC), the social preference test (SP), and the forced swim test (FST). Blood samples were taken $15 \mathrm{~min}$ after the end of the FST, and the animals were subsequently euthanized by transcardiac perfusion to process the brains for the immunohistochemical essays.

initiated. Before the start of the CUS protocol, the animals were tested in a battery of behavioral tasks, including the elevated plus maze (EPM), the open field/novel object test (OF/NO), the light and dark box (LD) and the circular corridor (CC), to determine their individual personality traits. To study the effect of individual differences on the impact of the stress protocol, the animals were matched according to their behavioral traits and body weights to ensure that the same personality traits and body weight distributions were obtained in each group. Subsequently, the matched rats were randomly assigned to one of the following experimental groups: (1) control (daily handling), (2) chronic unpredictable stress for 14 days, and (3) chronic unpredictable stress for 28 days.

Behavioral indexes of the impact of CUS were evaluated using a saccharin consumption test, a social preference test and a forced swim test performed on days zero, three and five, respectively, after exposure to CUS (Fig. 1). The body weight was measured the day before starting the stressinduction protocol and then every 3rd day throughout the stress period.

\subsection{Characterization of behavioral traits}

\subsubsection{Elevated plus maze}

Anxiety levels were evaluated using the EPM test (Herrero et al., 2006). Briefly, the test consists of two opposing open arms $(45 \mathrm{~cm} \times 10 \mathrm{~cm})$ perpendicular to two enclosed arms $(45 \mathrm{~cm} \times 10 \mathrm{~cm} \times 50 \mathrm{~cm})$ that extend from a central platform $(10 \mathrm{~cm} \times 10 \mathrm{~cm})$, elevated $65 \mathrm{~cm}$ above the floor. The rats were placed individually on the central platform and allowed to explore the maze for $5 \mathrm{~min}$. Their behavior was monitored using a video camera and analyzed with a computerized tracking system (EthoVision 3.1.16, Noldus IT, The Netherlands). The time spent in the open and closed arms, distance moved and transitions between the different arms were recorded.

\subsubsection{Open field and novel object reactivity}

Anxiety, exploration and activity-related behaviors were tested in the open field (OF) and novel object (NO) tests. The open field test consisted of a black circular arena (1 $\mathrm{m}$ in diameter and $40 \mathrm{~cm}$ high). The floor of the arena was divided into three zones: the outer zone, with a diameter of $1 \mathrm{~m}$; the inner zone, with a diameter of $75 \mathrm{~cm}$; and the center zone, with a diameter of $25 \mathrm{~cm}$. The light was adjusted to a level of 8-10 lx in the center of the pool. The animals were placed in the center of the arena, and the open field activity was tested over a 10 min period. Subsequently, a novel object was introduced into the center of the arena, and the animal's behavior was observed over the following $5 \mathrm{~min}$. The activity and behavior during the whole session was recorded with a video camera and automatically registered and analyzed with the EthoVision computerized tracking system (ColorPro 3.0.15, Noldus Information Technology, The Netherlands). The time spent in each of the virtually created zones (central zone, $25 \mathrm{~cm}$ diameter in the center of the apparatus; inner zone, $75 \mathrm{~cm}$ diameter in the center of the apparatus; and outer zone, $12.5 \mathrm{~cm}$ in the periphery of the apparatus next to the walls) was measured. Behavioral scoring of the time spent freezing and the time spent touching the novel object was done manually (Larsen et al., 2010).

\subsubsection{Light and dark box}

Anxiety levels were also evaluated using a modified light and dark box (Crawley and Goodwin, 1980) consisting of two equally sized chambers $(24 \mathrm{~cm}$ high $\times 25 \mathrm{~cm}$ wide $\times 33 \mathrm{~cm}$ long) that were connected by an $8 \mathrm{~cm} \times 8 \mathrm{~cm}$ opening. One chamber was black and covered by a lid (i.e., the dark box). The alternate chamber was white and remained uncovered during the test (i.e., the light box). A light positioned $165 \mathrm{~cm}$ above the light box provided illumination at a level of 500 lx. There was no appreciable illumination (i.e., $<2$ lx) in the dark box. The rats were placed in the center of the light box and allowed to freely explore the apparatus for $5 \mathrm{~min}$. The apparatus was cleaned with a $5 \%$ alcohol solution before testing each rat. Behaviors recorded during the test included: (1) the latency to enter the dark box, (2) the latency to return to the light box after entering the dark chamber, (3) the percent of time spent in the light box, (4) the number of chamber transitions, and (5) rearing in the light box.

\subsubsection{Circular corridor}

Exploration and activity-related behaviors were evaluated using a modified circular corridor (Rotllant et al., 2010; Piazza et al., 1989) consisting of a black circular arena $(80 \mathrm{~cm}$ in diameter $\times 34 \mathrm{~cm}$ high) with a cylinder placed in 
the center $(50 \mathrm{~cm}$ in diameter), forming a circular corridor $14 \mathrm{~cm}$ wide. The test was performed under infrared light, and the behavior during the entire session was recorded with a video camera that detected infrared light and was automatically registered and analyzed with the EthoVision computerized tracking system (Color-Pro 3.0.15, Noldus Information Technology, The Netherlands). During the 30 min test, the rat was placed inside the corridor and was allowed to move around the circular track between the walls. The following measures were taken in intervals of $5 \mathrm{~min}$ : (1) the total distance traveled, (2) the time spent in movement, and (3) the number of defecations.

\subsection{Chronic unpredictable stress}

As in our previous studies (Sandi et al., 2008; Larsen et al., 2010), the rats submitted to the CUS protocol were subjected each day to one of nine stressors in an unpredictable order and at an unpredictable time of the day for a period of either 2 or 4 weeks. The stressors included acoustic stimulation (78-115 dB 20-40 ms noise bursts; intertrial intervals ranged from 4 to $22 \mathrm{~s}$ and averaged $13 \mathrm{~s}$ : total duration of session: $15 \mathrm{~min}$ ), inverse light and dark cycle (over a $48 \mathrm{~h}$ period), exposure to overcrowding under a bright light (six rats in a standard home cage, 1000 (x, 2 h), inescapable foot shock (three foot shocks of $1 \mathrm{~mA}, 1 \mathrm{~s}, 1$ min intershock interval), an elevated platform (rats were placed on a platform $(20 \mathrm{~cm} \times 20 \mathrm{~cm})$ elevated $1 \mathrm{~m}$ above the ground for $2 \mathrm{~h}$ ), predator odor ( $1 \mathrm{~h}$ exposure to 2,5-dihydro-2,4,5-trimethylthiazoline, a synthetic compound originally isolated from fox feces; Fendt and Endres, 2008), bright light and water deprivation (1000 lx, $30 \mathrm{~min})$, food deprivation (24 h), and forced swim stress (15 min). The stressed pairs of rats were housed separated by transparent plastic wall, which allowed visual and olfactory contact between them. Nonstressed control rats were housed 2 per cage without the separator and were briefly handled every day during the stress period.

\subsection{Behavioral testing}

\subsubsection{Saccharin consumption}

A saccharin consumption test was performed on days $0,7,14$, 21 and 28 of the stress period. The rats were given a free choice between two bottles, one containing normal drinking water and the other containing a $0.02 \%$ saccharin solution. The bottles were available for $12 \mathrm{~h}$. The saccharin intake was calculated as the amount consumed in grams per $100 \mathrm{~g}$ body weight. The saccharin preference was calculated as saccharin intake/total fluid intake (water + saccharin) (Larsen et al., 2010).

\subsubsection{Social preference}

On the third day after CUS, the social preference test was conducted in a rectangular, three chambered box (a center compartment of $20 \mathrm{~cm} \times 35 \mathrm{~cm} \times 35 \mathrm{~cm}$ with a left and a right compartment of $30 \mathrm{~cm} \times 35 \mathrm{~cm} \times 35 \mathrm{~cm}$ ) fabricated from opaque gray polycarbonate. The dividing walls had retractable doorways allowing access to each chamber. The test rat was placed in the middle chamber and allowed to explore the entire apparatus for $10 \mathrm{~min}$. Each of the two side chambers contained an empty wire cage. The wire cages were $10 \mathrm{~cm}$ in height, with a bottom diameter of $9 \mathrm{~cm}$ and bars spaced $1 \mathrm{~mm}$ apart. A weighted plastic cone was placed on the top of each cage to prevent climbing by the test rats. Four sets of wire cages were used during the experiment, and all of the cages were washed with water and dried properly between each use.

For habituation to the wire cage, each novel pre-pubertal male rat used in the social interaction test had been previously placed in the wire cage in the apparatus without the test rat for 5 min on 3 consecutive days preceding the social test. On the day after the last habituation session, a test rat was placed in the center compartment and allowed to explore the entire apparatus for $10 \mathrm{~min}$. An unfamiliar rat was placed in one of the wire cages located on either side of the social test box during the 10 min session. A rectangular colored object was placed in the other wire cage on the other side of the box. The location of the stranger and the object in the left and right sides of the chamber was counterbalanced for different animals. Placing the strange rat in a wire cage prevented direct physical contact between the rats and ensured that the social approach was only initiated by the test rat. The time spent sniffing each wire cage was videorecorded and manually scored to evaluate the level of preference for the unfamiliar rat as compared to the object. The entire apparatus was cleaned with water and dried thoroughly between each tested rat.

\subsubsection{Forced-swim test}

On day 5 after the CUS, the animals were subjected to a forced swim test (Porsolt et al., 1977) to evaluate potential differences in depression-like behavior between the stressed and control animals (Castro et al., 2010; Larsen et al., 2010). Briefly, the animals were individually placed in a plastic beaker ( $25 \mathrm{~cm}$ in diameter, $46 \mathrm{~cm}$ deep) containing $30 \mathrm{~cm}$ of water $\left(25 \pm 1^{\circ} \mathrm{C}\right)$ for $5 \mathrm{~min}$. Their behavior was recorded with a video camera, and the time spent immobile (making only those movements necessary to keep the snout above the water), swimming, climbing and diving was quantified using a computer program (The Observer 5.0.25, Noldus IT, 2003). Although the standard use of the forced swim test when applied for psychopharmacology purposes typically includes a first 15-min induction session followed by a 5 -min testing session $24 \mathrm{~h}$ later, our study - not focusing on drug effects but on the assessment of the impact of the prior stress experience - only included a single 15-min test session. The reason for this procedure was two-fold. First, as just indicated, to serve as a readout of the impact of exposure to different lengths of repeated stress in different animal groups. Second, to act as a stress challenge for the immediate evaluation of ERK activation without the confounding of potential memory factors that could be manifested on a second, repetition test.

\subsection{Histological procedures}

Fifteen minutes after the end of the forced-swim test, the animals were anesthetized with isoflurane and transcardially perfused using a $0.9 \%$ saline solution followed by a fixative solution of paraformaldehyde $4 \%$ in phosphate buffered saline ( $\mathrm{PBS}, \mathrm{pH}=7.5$ ). After perfusion-fixation, the brains were removed and post-fixed in the same solution for $4 \mathrm{~h}$. Next, 
$50 \mu \mathrm{m}$ thick coronal sections were cut on a vibratome (VT 1000S; Leica, Glattbrugg, Switzerland) and stored at $4{ }^{\circ} \mathrm{C}$ in PBS. Brain ERK1/2 activity was assayed by staining for pERK1/2.

An alternate series of 1 in 10 sections was processed "free-floating" for immunohistochemical visualization of PERK $1 / 2$. Briefly, the sections were incubated with $10 \%$ $\mathrm{H}_{2} \mathrm{O}_{2}$ in PBS for 10 min to block the endogenous peroxidase activity. After washing in PBS $(3 \times 10 \mathrm{~min})$, the sections were treated for $1 \mathrm{~h}$ with $10 \%$ normal donkey serum (NDS, Jackson ImmunoResearch Laboratories, West Grove, PA) in PBS with 0.2\% Triton-X100 (Sigma-Aldrich, St. Louis, MO). After washing in PBS, the sections were incubated overnight at $4{ }^{\circ} \mathrm{C}$ with rabbit polyclonal anti pERK1/2 lgG, which stains the activated form of ERK1/2 (1:500; Cell Signaling Technology). PBS containing $0.2 \%$ Triton-X100 and 5\% NDS (PBST) was used to dilute the primary and secondary antibodies. After washing in PBS, the sections were incubated for 120 min with goat antirabbit IgG (1:2000; Jackson ImmunoResearch Laboratories). After washing in PBS, the sections were incubated for $1 \mathrm{~h}$ with the avidin-biotin-peroxidase complex (ABC; A 1:200, B 1:200; Vector Laboratories, Peterborough, UK), which was prepared 30 min prior to incubation in PBS. After washing in PBS, color development was achieved by incubating the sections in 3,3'-diaminobenzidine tetrahydrochloride (DAB; $0.5 \mathrm{mg} / \mathrm{ml}$, Sigma-Aldrich) for $15 \mathrm{~min}$.

\subsection{Image analysis}

The levels of brain ERK1/2 activity (phosphorylated ERK1/2; pERK1/2) were estimated by densitometric analyses of the staining in the basolateral, central, medial and basomedial nuclei of the amygdala, the CA1, the CA3 and the dentate gyrus of the hippocampus, and the corpus callosum. Digital pictures were taken from coronal sections using a Northern Light transilluminator (model R95) and a Photometrics Coolsnap camera (Roper scientific) system. Protein expression levels were semi-quantitatively determined by measuring the optical densities and the number of pixels in the defined areas with Analysis Pro 5.0. The mean pERK1/2 protein expression levels per area are expressed in arbitrary units (number of pixels per optic density). All of the compared samples were processed in the same immunohistochemical assay to avoid inter-assay variability. The optical density data for all of the brain regions were normalized to the optical density obtained for the corpus callosum.

\subsection{Corticosterone analyses}

Corticosterone responses to stress were evaluated in plasma samples obtained 15 min after testing in the forced swim test. Plasma was obtained from blood collected cardiac puncture immediately after anesthesia and just before perfusion. Three hundred microliters of blood was taken using a capillary tube containing heparin (Sarted, Germany). The blood samples were centrifuged, and the plasma was stored at $-20{ }^{\circ} \mathrm{C}$ until the assay. The plasma corticosterone levels were quantified using the Correlate-EIA Corticosterone Enzyme Immunoassay Kit (Assay Design, MI, USA) according to the manufacturer's instructions. The intra-assay coefficient of variance was $8 \%$.

\subsection{Statistical analyses}

The SPSS 13.0 (SPSS, Chicago, IL) statistical package was used for the statistical analyses. The normality and homogeneity of variance of the data were tested, and the adjusted statistics were used as required.

\subsubsection{Factorial analyses}

Two-fold exploratory factor analyses were used to obtain a continuous interval scale score by using principal components as an extraction method and the varimax rotation with the Kaiser normalization rotation (Doremus et al., 2006). Individual factor scores from the parameters of the EPM, OF/NO, LD and CC were calculated for each subject based on the relative weight and orientation (eigenvalues) of the loading of the parameters for each obtained factor. The scores were generated using a $Z$ distribution, where a value of 0 corresponds to the mean, and the values were expressed in terms of their standard deviations. The animals were matched based on their scores for the different factors and classified into different experimental groups to yield groups with similar personality-like traits. In addition, the data from the factorial analyses were used to investigate the modulatory effect of the factor score differences on the stress effects investigated. The animals were classified into groups such that the group scores were either above or below the overall mean for each of the factors. Using the extreme low and high values of the continuous scores obtained from the factor analyses, categorical dichotomous scores were obtained by classifying the subjects into groups of low and high scores for each factor; the subjects with scores within \pm 0.25 standard deviations from the mean were excluded from this classification.

\subsubsection{Parametric statistics}

The general effects of chronic stress on body weight gain, behavioral indexes, corticosterone response, and ERK1/2 activity were analyzed with one-way ANOVAs followed by post hoc comparisons. Sequential three-way ANOVAs followed by simple effects analyses were used to assess the interaction effect of chronic stress conditions and the levels of anxiety exploration traits $(3 \times 2 \times 2$, whenever analyses including the three personality-like traits were not significant; analyses of different combinations of two traits followed $2 \times 2 \times 2$ ) on body weight gain, behavioral indices, corticosterone response, and ERK1/2.

\section{Results}

\subsection{Characterization of personality traits}

To characterize the animals according to their personality traits, factorial analyses were applied separately to a range of extracted parameters from the EPM, OF/NO, LD and CC tests, which were conducted before the animals' exposure to the CUS (Table S1). Then, an overall factor analysis was performed on the extracted factors, and this analysis revealed three factors, which were termed activity, anxiety and exploration according to the parameters that defined them (Table S1). The data obtained from the animals' scores for each of these factors were used to classify the animals 
into dichotomized variables for each behavioral trait (i.e., activity, anxiety and exploration). Thus, the animals were classified as follows according to whether their score was above or below the mean for each factor: low (LL) or high (HL) locomotion, low (LA) or high (HA) anxiety, and low (LE) or high (HE) exploration. The orthogonality of the personality traits was confirmed by comparing the mean scores for each variable (see Fig. S1 for an example of no differences in the exploration trait values between the animals classified as either LA or HA). Throughout the study, factorial ANOVAs with the three personality traits revealed a lack of significant interaction (n.s.). Further factorial ANOVAs that were performed on combinations of two behavioral traits as the factors did not yield statistical significance, except when 'anxiety' and 'exploration' traits were combined. These two behavioral traits were also previously identified to explain variance in stressful learning (Salehi et al., 2010). Therefore, this factorial ANOVA will subsequently be referred to in the text.

\subsection{Effects of CUS and personality traits on body weight}

One-way ANOVAs were performed to assess the impact of two and four weeks of CUS on body weight. The ANOVA showed a significant effect of chronic stress on body weight gain during the CUS period $[F(2,123)=34.13 ; p=0.001]$. Post hoc analyses showed a progressive decrease after two $(p=0.001)$ and four weeks $(p=0.001)$ of CUS compared to the control group. Moreover, the group receiving four weeks of CUS differed from the group receiving two weeks of CUS $(p=0.05)$ (Fig. 2A). Three-way ANOVAs were performed to assess the impact of CUS, and anxiety and exploration traits on body weight. ANOVA showed a significant interaction effect on body weight gain $[F(2,114)=3.60 ; p=0.03]$. A further simple effect analysis indicated a decrease in body weight in the groups receiving two weeks of CUS compared to the control group in all of the personality profiles except the 'low anxiety and low exploration' profile (LA/HE $p=0.02$; HA/LE $p=0.001$; HA/HE $p=0.001$ ). Additionally, only the 'low anxiety and low exploration' $(p=0.03)$ and 'high anxiety and high exploration' $(p=0.001)$ personality profiles showed significant decreases in body weight in the group receiving four weeks of CUS compared to the group receiving two weeks of CUS (Fig. 2B).

\subsection{Effects of CUS and personality traits on depression-like behaviors}

One-way ANOVAs were performed to assess the impact of two and four weeks of CUS on depression-like behaviors, including anhedonia measured by saccharin intake in a preference task, social avoidance measured by the interaction with a conspecific juvenile animal versus an object in a preference task and immobility in the forced swim test. Three-way ANOVAs were performed to assess the impact of CUS, anxiety and exploration traits on these depression-like behaviors.

\subsubsection{Effects of CUS and personality traits on saccharin consumption}

An ANOVA showed a significant effect of chronic stress on anhedonia measured by saccharin intake in a preference task $[F(2,139)=8.16 ; p=0.001]$. Post hoc analyses showed a decrease in saccharin intake both in animals receiving two ( $p=0.001)$ or four $(p=0.02)$ weeks of CUS compared to the control group. This result was observed both when analyzed as average intake (Fig. 3A) and as consumption across weekly testing (Fig. 3B). A factorial ANOVA showed non-significant interactions of the personality traits with anhedonia measured by saccharin intake in a preference task (data not shown).

\subsubsection{Effects of CUS and personality traits on social preference}

An ANOVA showed a significant effect of chronic stress on social avoidance measured by the interaction with an animal versus an object in the preference task $[F(2,128)=33.38$;
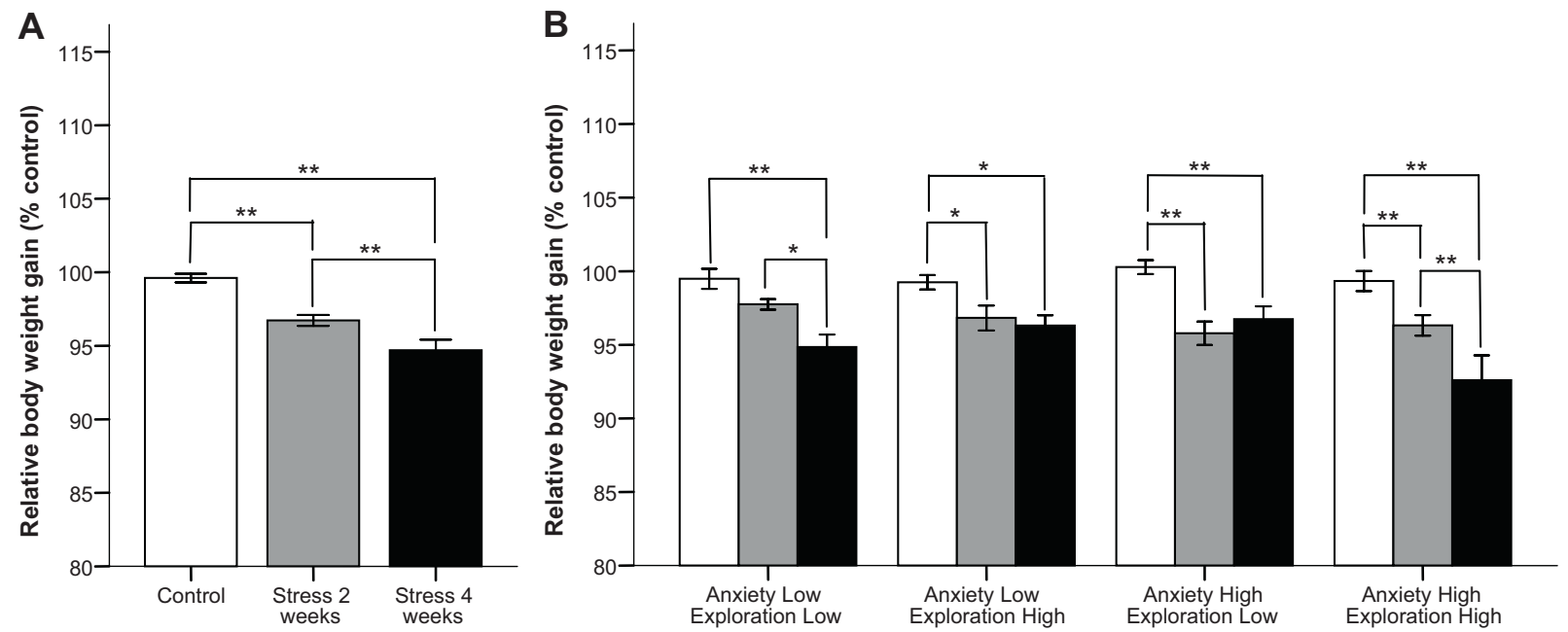

Figure 2 A. The effects of chronic unpredictable stress on body weight gain. Control group: $n=56,2$ weeks of CUS: $n=42,4$ weeks of CUS: $n=28$. B. Interaction effect of chronic unpredictable stress and personality traits on body weight gain. Control group: LA/LE $n=10$, LA/HE $n=14$, HA/LE $n=16$, HA/HE $n=16 ; 2$ weeks of CUS: LA/LE $n=10$, LA/HE $n=12$, HA/LE $n=6$, HA/HE $n=14 ; 4$ weeks of CUS: LA/LE $n=7$, LA/HE $n=6$, HA/LE $n=5$, HA/HE $n=10 .{ }^{*} p<0.05,{ }^{* *} p<0.01$. 

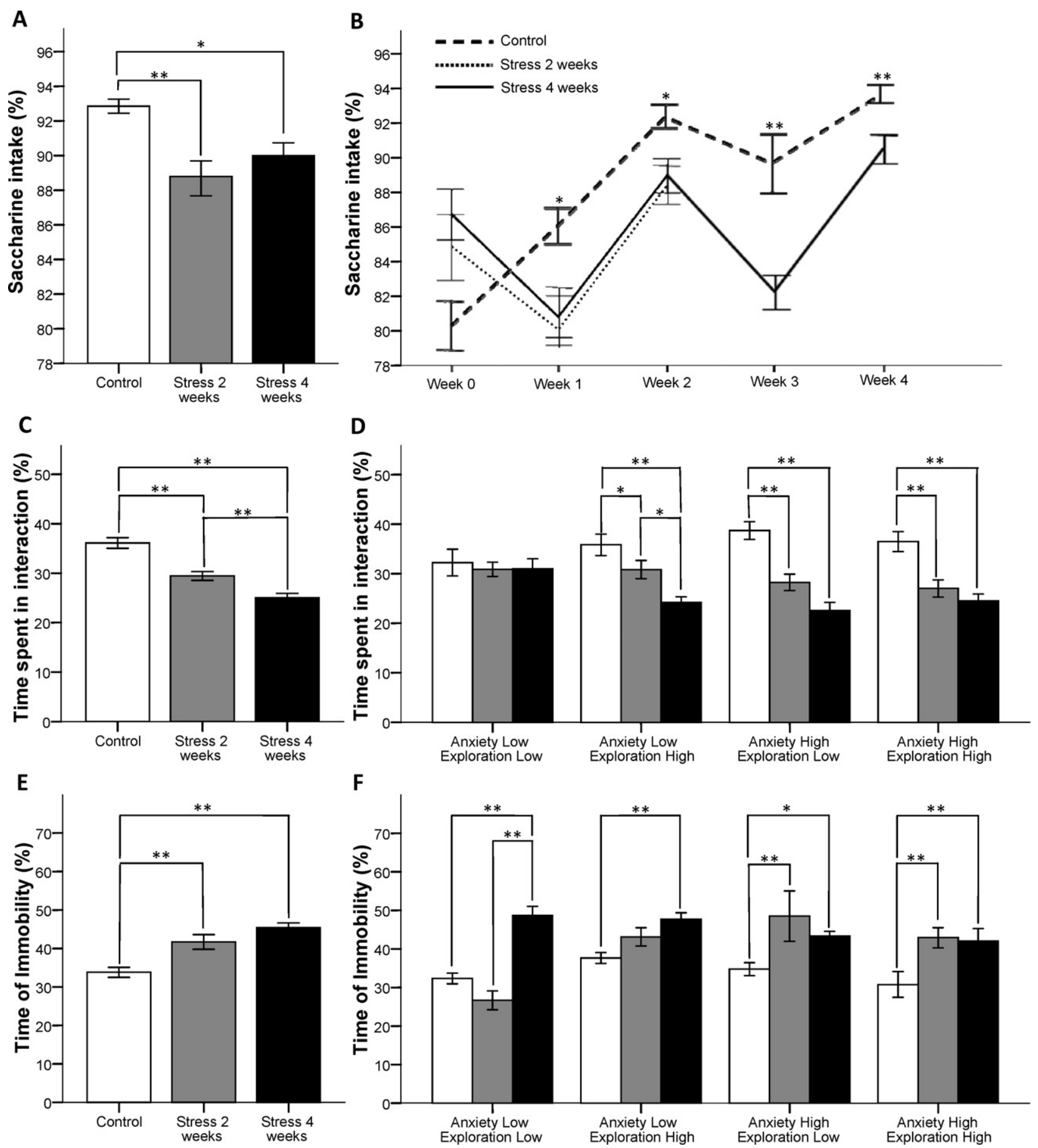

Figure 3 The effects of chronic unpredictable stress and personality traits on different depression-like behaviors. A,B. The effects of chronic unpredictable stress on anhedonia measured by the saccharin intake in a preference task. Control group: $n=56,2$ weeks of CUS: $n=43$, 4 weeks of CUS: $n=43$. C. The effects of chronic unpredictable stress on social avoidance measured by the interaction with another animal in a preference task. Control group: $n=50,2$ weeks of CUS: $n=42,4$ weeks of CUS: $n=39$. D. The interaction effect of chronic unpredictable stress and personality traits on social avoidance measured by the interaction with another animal in a preference task. Control group: LA/LE $n=9$, LA/HE $n=13$, HA/LE $n=13$, HA/HE $n=15$; 2 weeks of CUS: LA/LE $n=12$, LA/HE $n=13$, HA/LE $n=5$, HA/HE $n=12 ; 4$ weeks of CUS: LA/LE $n=7$, LA/HE $n=10$, HA/LE $n=11$, HA/HE $n=11$. E. The effects of chronic unpredictable stress on depression-like behavior measured by immobility in the forced swim test. Control group: $n=39,2$ weeks of CUS: $n=37,4$ weeks of CUS: $n=38$. F. The interaction effect of chronic unpredictable stress and personality traits on depression-like behavior measured by immobility in the forced swim test. Control group: LA/LE $n=7$, LA/HE $n=11$, HA/LE $n=8$, HA/HE $n=13$; 2 weeks of CUS: LA/LE $n=5$, LA/HE $n=12$, HA/LE $n=6$, HA/HE $n=14 ; 4$ weeks of CUS: LA/LE $n=10$, LA/HE $n=9$, HA/LE $n=9$, HA/HE $n=10$. The values show the mean + SEM; ${ }^{*} p<0.05,{ }^{* *} p<0.01$. 
$p=0.001]$, and post hoc analyses showed a progressive decrease in the interaction with the conspecific juvenile after two $(p=0.001)$ and four $(p=0.001)$ weeks of CUS (Fig. 3B). A factorial ANOVA showed a significant interaction effect on social avoidance measured by the interaction with another animal in a preference task $[F(2,119)=3.61$; $p=0.03]$, and a simple effect analysis demonstrated a decrease in the interaction with another animal in animals receiving two (LA/HE $p=0.04$; HA/LE $p=0.001 ; \mathrm{HA} / \mathrm{HE}$ $p=0.001$ ) or four (LA/HE $p=0.001$; HA/LE $p=0.001$; HA/ HE $p=0.001$ ) weeks of CUS compared to the control group for all of the personality profiles except the 'low anxiety and low exploration' profile (Fig. 3C).

\subsubsection{Effects of CUS and personality traits on the forced swim test}

An ANOVA showed a significant effect of chronic stress on depression-like behavior measured by immobility in the forced swim test $[F(2,111)=16.03 ; p=0.001]$, and post hoc analyses showed an increase in the time spent immobile in animals receiving either two $(p=0.001)$ or four $(p=0.001)$ weeks of CUS compared to the control group (Fig. 3C). A factorial ANOVA showed a significant interaction effect on depression-like behavior measured by immobility in the forced swim test $[F(2,102)=3.48 ; p=0.03]$, and simple effect analyses showed an increase in the time spent immobile in the animals receiving two weeks of CUS compared to the control group only in the 'high anxiety and low exploration' $(p=0.001)$ and 'high anxiety and high exploration' $(p=0.001)$ personality profiles (Fig. 3E).

\subsection{Effects of CUS and personality traits on the corticosterone response}

One-way ANOVAs were performed to assess the impact of two and four weeks of CUS on the corticosterone response to acute stress, which was analyzed $15 \mathrm{~min}$ after the forced swim test. An ANOVA showed a significant effect of chronic stress on the corticosterone response $[F(2,72)=9.09$; $p=0.001$ ], and post hoc analyses showed a gradual increase in the corticosterone response both in animals receiving two weeks $(p=0.02)$ or four weeks $(p=0.001)$ of CUS compared to the control group (Fig. 4A). Factorial ANOVAs were performed to assess the impact of CUS and anxiety and exploration traits on the corticosterone response. An ANOVA showed a marginal interaction effect on the corticosterone response to the acute stress induced by the forced swim test $[F(2,67)=2.58 ; p=0.08]$, and simple effect analyses indicated an increase in the corticosterone response in the animals receiving two weeks of CUS compared to the control group only in animals with the 'high anxiety and low exploration' ( $p=0.001$ ) personality profile. Additionally, an increase in the corticosterone response in animals receiving four weeks of CUS compared to animals receiving two weeks of CUS was only found in the 'low anxiety and low exploration' $(p=0.04)$ and 'high anxiety and high exploration' $(p=0.04)$ personality profiles (Fig. 4B).

\subsection{Effects of CUS and personality traits on brain ERK1/2 activity}

One-way ANOVAs were performed to assess the impact of two and four weeks of CUS on brain ERK activity, and factorial ANOVAs were performed to assess the impact of CUS and anxiety and exploration traits on brain ERK activity. An ANOVA showed no significant effect of chronic stress on pERK levels in the corpus callosum, which was used as a negative control for pERK immunohistochemical signal (n.s.; data not shown).

\subsubsection{Effects of CUS and personality traits on ERK1/2} activity in the amygdala

ANOVAs showed a significant effect of chronic stress on pERK levels in the basolateral $[F(2,91)=4.80 ; p=0.01]$ and
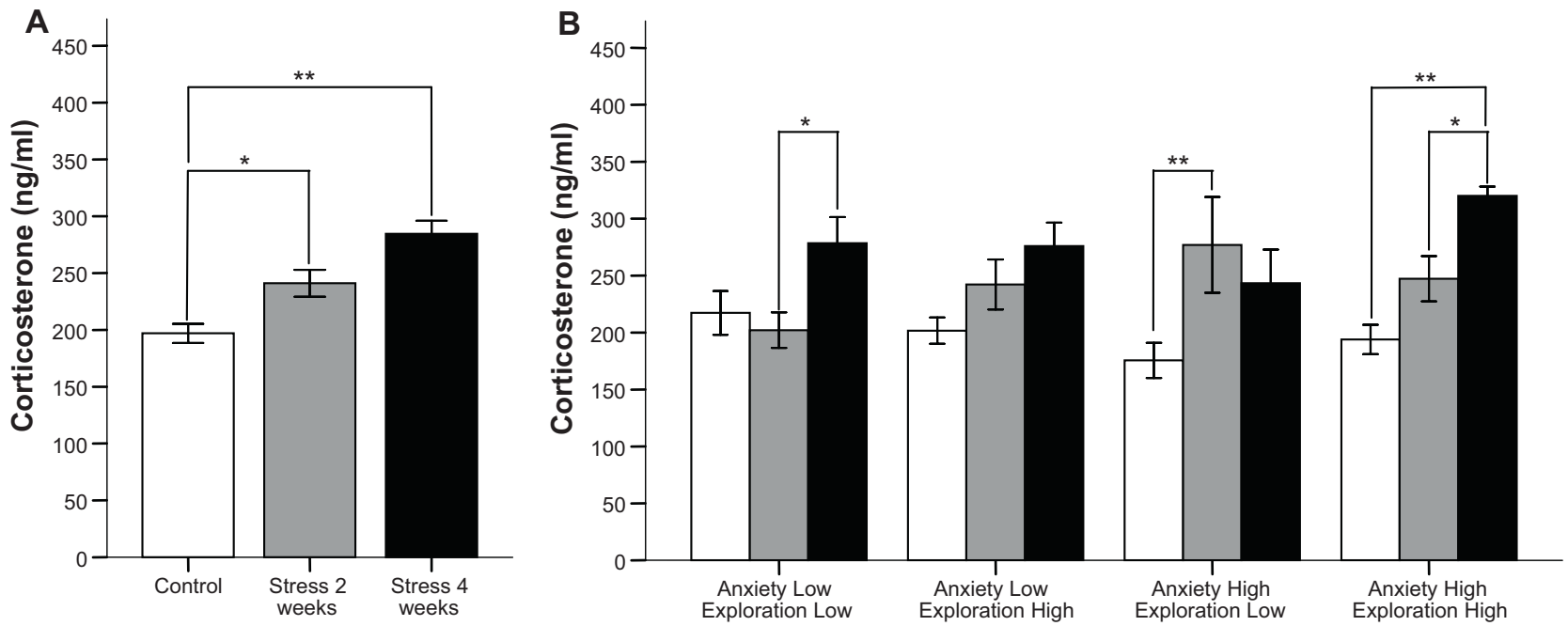

Figure 4 A. The effects of chronic unpredictable stress on corticosterone levels after 15 min of forced swimming. Control group: $n=22$, 2 weeks of CUS: $n=39,4$ weeks of CUS: $n=14$. B. The interaction effect of chronic unpredictable stress and personality traits on corticosterone levels after 15 min of forced swimming. Control group: LA/LE $n=6$, LA/HE $n=4$, HA/LE $n=7$, HA/HE $n=6$; 2 weeks of CUS: LA/LE $n=10$, LA/HE $n=13$, HA/LE $n=6$, HA/HE $n=11 ; 4$ weeks of CUS: LA/LE $n=4$, LA/HE $n=4$, HA/LE $n=4$, HA/HE $n=4$. The data show the mean + SEM; ${ }^{*} p<0.05,{ }^{* *} p<0.01$. 

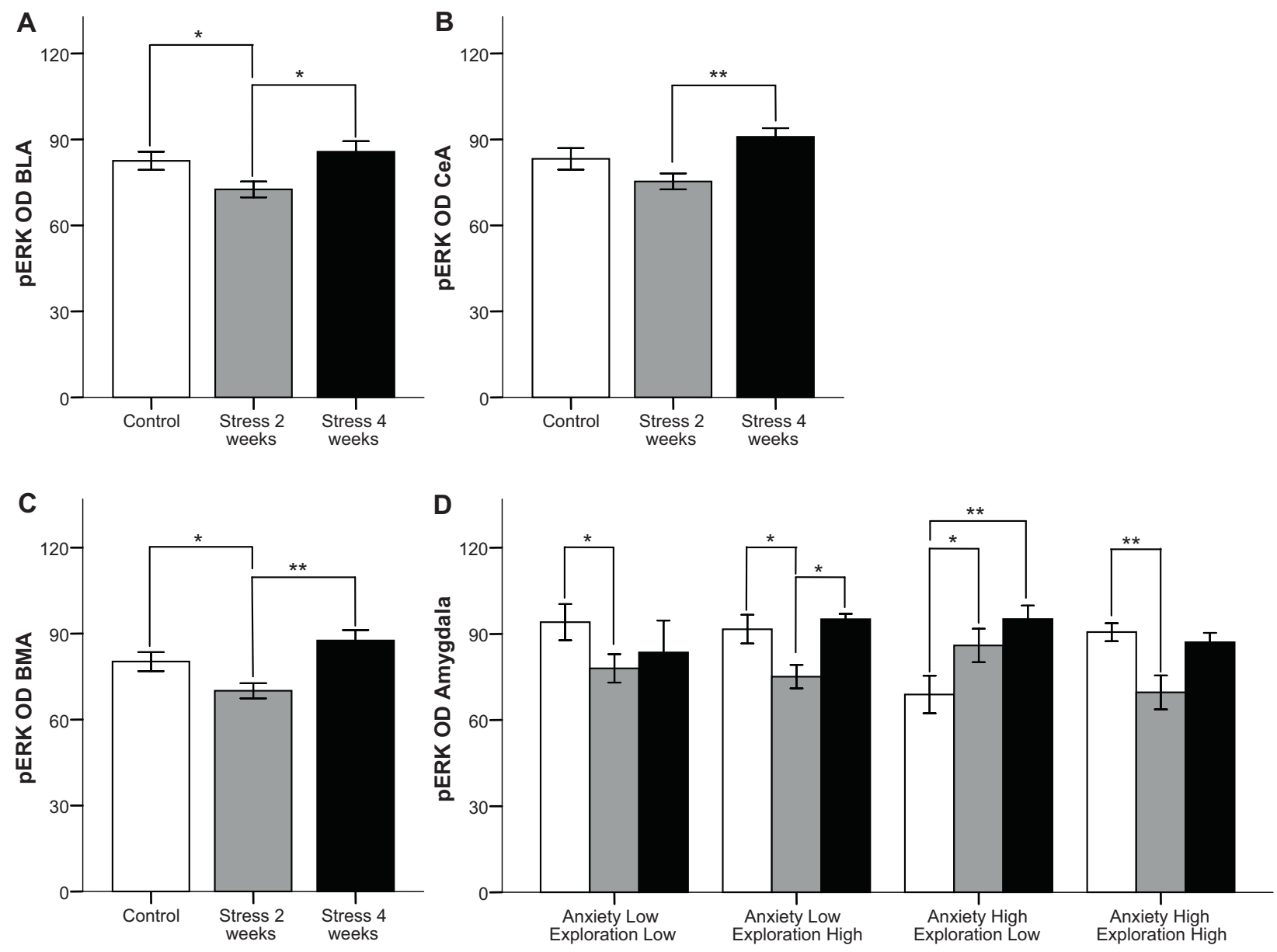

Figure 5 A. The effects of chronic unpredictable stress on ERK activity in the basolateral amygdala. Control group: $n=30,2$ weeks of CUS: $n=46,4$ weeks of CUS: $n=18$. B. The effects of chronic unpredictable stress on ERK activity in the central amygdala. Control group: $n=30$, 2 weeks of CUS: $n=46,4$ weeks of CUS: $n=18$. C. The effects of chronic unpredictable stress on ERK activity in the basomedial amygdala. Control group: $n=29,2$ weeks of CUS: $n=45$, 4 weeks of CUS: $n=18$. D. The interaction effect of chronic unpredictable stress and personality traits on ERK activity in the amygdala. Control group: LA/LE $n=6, \mathrm{LA} / \mathrm{HE} n=6, \mathrm{HA} / \mathrm{LE} n=9$, HA/ HE $n=9 ; 2$ weeks of CUS: LA/LE $n=14$, LA/HE $n=13$, HA/LE $n=6$, HA/HE $n=13 ; 4$ weeks of CUS: LA/LE $n=5$, LA/HE $n=4$, HA/LE $n=5$, $\mathrm{HA} / \mathrm{HE} n=4$. The data show the mean + SEM; ${ }^{*} p<0.05,{ }^{* *} p<0.01$.

basomedial $[F(2,89)=7.38 ; p=0.001]$ amygdala, and post hoc analyses showed a "U-shaped" effect of stress in both of these regions. Thus, after two weeks of CUS, there was a significant decrease of pERK levels compared to controls ( $p=0.05$ and $p=0.04$, respectively) and animals receiving four weeks of CUS ( $p=0.02$ and $p=0.001$, respectively) (Fig. 5A and C). An ANOVA also showed a significant effect of chronic stress on pERK levels in the central amygdala $[F(2,91)=4.96 ; p=0.01]$, and post hoc analyses specified an increase in $\mathrm{PERK}$ in the central amygdala after four weeks of CUS compared to two weeks of CUS ( $p=0.01)$ (Fig. 5B).

$A$ factorial ANOVA indicated a significant interaction effect on pERK levels in the amygdala (a compound measure for this nucleus was used because the data for individual nuclei depicted similar results) $(F(2,82)=3.51 ; p=0.03)$. In the 'high anxiety and low exploration', the groups receiving two $(p=0.05)$ or four $(p=0.001)$ weeks of CUS showed an increase in pERK levels compared to the control group. This is at odds with the other three behavioral profiles, that in all cases showed a decrease in pERK $1 / 2$ levels after two weeks of CUS as compared to controls (LA/LE $p=0.05$; LA/HE $p=0.04$;
HA/HE $p=0.001$ ) while pERK1/2 levels after four weeks of CUS did not differ from control (n.s.) (Fig. 5D).

\subsubsection{Effects of CUS and personality traits on ERK $1 / 2$ activity in the hippocampus}

An ANOVA showed no significant effect of chronic stress on pERK levels in the CA1 area of the hippocampus (Fig. 6A). However, an ANOVA showed a significant effect of chronic stress on pERK levels in the CA3 area of the hippocampus $[F(2,93)=3.66 ; p=0.03]$, and post hoc analyses indicated an increase in pERK levels after four weeks of CUS compared to two weeks of CUS ( $p=0.03$ ) (Fig. 6B). Additionally, an ANOVA showed a significant effect of chronic stress on $\mathrm{pERK}$ levels in the hippocampal dentate gyrus $[F(2,93)=3.98 ; p=0.02]$, and post hoc analyses showed a decrease in pERK levels in the group receiving two weeks of CUS compared to the control group $(p=0.05)$ (Fig. 6C).

An ANOVA showed a significant interaction effect of the anxiety and exploration traits on pERK levels in the hippocampus (a compound measure for this area was used because the data for the different hippocampal regions depicted 

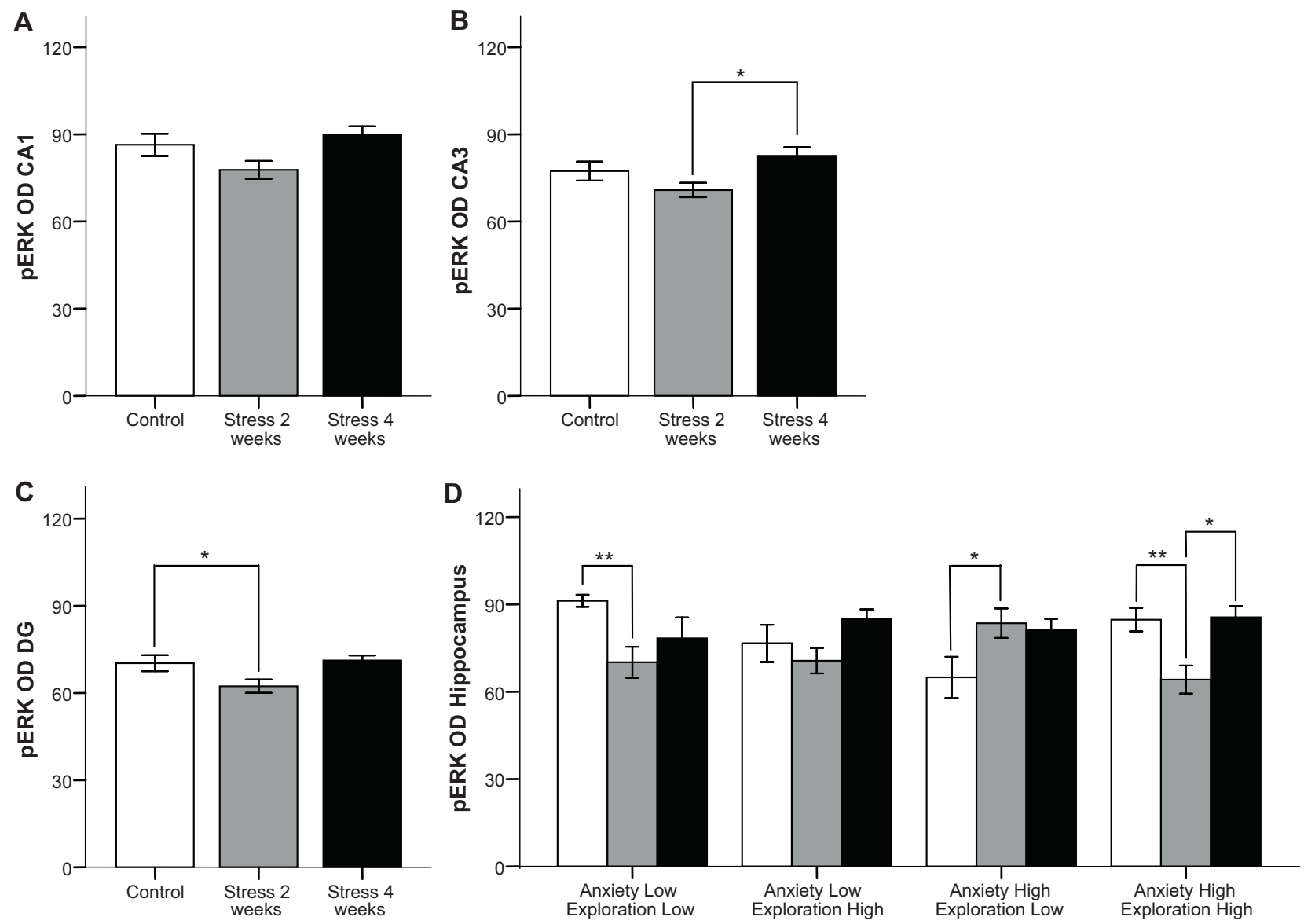

Figure 6 A. The effects of chronic unpredictable stress on ERK activity in the hippocampal CA1 area. Control group: $n=30,2$ weeks of CUS: $n=48,4$ weeks of CUS: $n=18$. B. The effects of chronic unpredictable stress on ERK activity in the hippocampal CA3 area. Control group: $n=30,2$ weeks of CUS: $n=48,4$ weeks of CUS: $n=18$. C. The effects of chronic unpredictable stress on ERK activity in the hippocampal dentate gyrus. Control group: $n=30,2$ weeks of CUS: $n=48,4$ weeks of CUS: $n=18$. D. The interaction effect of chronic unpredictable stress and personality traits on ERK activity in the hippocampus. Control group: LA/LE $n=6, \mathrm{LA} / \mathrm{HE} n=6, \mathrm{HA} / \mathrm{LE} n=9$, HA/HE $n=9 ; 2$ weeks of CUS: LA/LE $n=14$, LA/HE $n=13$, HA/LE $n=7$, HA/HE $n=14 ; 4$ weeks of CUS: LA/LE $n=5$, LA/HE $n=4$, HA/LE $n=5, \mathrm{HA} / \mathrm{HE} n=4$. The data show the mean + SEM; ${ }^{*} p<0.05,{ }^{* *} p<0.01$.

similar results) $[F(2,84)=6.41 ; p=0.001]$. Further simple effect analyses showed a decrease in pERK levels in the 'low anxiety and low exploration' and 'high anxiety and high exploration' personality profiles in the group receiving two weeks of CUS compared to the control group (LA/LE $p=0.01$; $\mathrm{HA} / \mathrm{HE} p=0.001)$. Simple effect analyses showed an increase in pERK levels in the 'high anxiety and low exploration' personality profile in the group receiving two weeks of CUS compared to the control group $(p=0.01)$. In addition, in control animals, the 'high anxiety and low exploration personality' profile displayed lower pERK levels than the 'low anxiety and low exploration' $(p=0.001)$ personality profile (Fig. 6D).

\section{Discussion}

Chronic stress is a known risk factor for the development of depression in the general population (McEwen, 2004; de Kloet et al., 2005; Sandi and Richter-Levin, 2009). However, all individuals are not equally susceptible to the adverse effects of stress; some individuals are resistant to stress, whereas others show a high vulnerability to stress (Southwick et al., 2005; Feder et al., 2009). Both genetic factors and factors related to the individual's life history have been implicated in this susceptibility variation (Southwick et al., 2005; Feder et al., 2009; Sandi and Richter-Levin, 2009). These factors determine, among other things, an individual's personality traits, coping styles and other behavioral and physiological characteristics that have been hypothesized to be involved in the link between stress and psychopathology. Emerging evidence indicates that anxiety (Sandi and Richter-Levin, 2009) and exploration (Lara and Akiskal, 2006) traits are linked to stress-induced depression (Sandi et al., 2008; Revest et al., 2009; Fuss et al., 2010) and high glucocorticoid reactivity (Tyrka et al., 2008). A key challenge in this field is to determine the moderating effects of personality factors on an individual's vulnerability to depression and the neurobiological pathways underlying this differential vulnerability to stress. The aim of this study was to identify the behavioral traits and/or profiles that are associated with vulnerability and resilience to stressinduced depression-like behaviors and to evaluate HPA axis activity and $\mathrm{pERK} 1 / 2$ reactivity. To evaluate the differential vulnerability linked to specific personality profiles that may 
emerge with different levels of stress exposure, rats were exposed to either two or four weeks of CUS.

We first focused our analyses on the impact of progressive exposure to stress in the entire population. Thus, we evaluated the data from all of the animals submitted to CUS regardless of their personality profiles. All of the physiological (i.e., body weight gain, plasma corticosterone to acute stress), behavioral (i.e., tests for different depression-like behaviors) and neurobiological (i.e., pERK activity in the amygdala) parameters evaluated showed significant alterations after exposure to 2 weeks of stress. Except for pERK activity in the amygdala, which returned to control levels after a longer stress exposure, physiological and behavioral alterations became more pronounced with 4 weeks of stress exposure. However, PERK activation in the hippocampus (except for the dentate gyrus, which showed a decrease after two weeks of CUS) was not affected after 2 weeks of chronic stress. This apparent resistance of the hippocampus to the accumulated effects of stress exposure is consistent with morphological studies showing that changes in hippocampal dendritic morphology observed after chronic stress require several weeks to develop, with pyramidal cell dendritic atrophy observed after 21 but not after 14 days of repeated stress exposure (Magariños and McEwen, 1995). More generally, these results are consistent with a growing body of data indicating that the amygdala is more vulnerable than the hippocampus to the effects of stress. This difference is reflected by a faster reactivity of the structural features of the amygdala to the effects of stress exposure; 10 days of immobilization stress was shown to be sufficient to induce dendritic hypertrophy and spine formation in the basolateral amygdala (Mitra et al., 2005). Additionally, the difference is reflected by a slower ability of the amygdala to recover from chronic stress; even after 21 days of stress-free recovery from a previous chronic immobilization stress, a persistent increase in dendritic arborization in the basolateral amygdala spiny neurons was observed, whereas hippocampal CA3 atrophy had completely recovered by this time point (Vyas et al., 2004). Only a 10-day stress-free period was sufficient to reverse hippocampal CA3 atrophy (Conrad et al., 1999).

In addition to a robust (in terms of statistical significance) response in $\mathrm{pERK}$ activation in the amygdala as compared to the hippocampus after two weeks of stress, our results indicate a highly similar time-dependent, U-shaped pattern of ERK1/2 activity in these two structures. Interestingly, in the basolateral amygdala, the ERK1/2 signaling pathway has been shown to be critical for mediating stress effects on hippocampal synaptic plasticity (Yang et al., 2008). This similarity in the pattern of ERK1/2 activation in these two structures might appear surprising given the contrasting patterns of structural remodeling frequently reported for chronic stress effects in the hippocampus (dendritic atrophy in the CA3 area) and the amygdala (dendritic hypertrophy in the BLA) (Vyas et al., 2002). However, these opposing morphological effects were observed when immobilization stress was used, whereas a CUS model that was similar to the one used in our study was found to induce similar dendritic atrophy-like effects in the hippocampus and BLA (Vyas et al., 2002), which is consistent with the similar pattern of pERK activation observed in these two structures in our study. In contrast, the U-shaped pattern of activation observed in our study might suggest a habituation to the repeated exposure of stress, which has been speculated in the case of the BLA (Vyas et al., 2006), because no effects of stress were observed following 21 days of restraint stress in the levels of polysialylated neural cell adhesion molecule (PSA-NCAM), a key mediator of structural plasticity (Cordero et al., 2005). Our results on pERK levels, particularly in the amygdala, support the habituation hypothesis, particularly when taking into account the differences in the pattern of activation related to the personality profiles and their link with the behavioral and physiological results (see below). The habituation of behavioral or plasticity markers to repeated stress exposure might depend on many factors, such as individual differences, as suggested by our results, or the intensity of the stressor used (Vyas et al., 2006).

However, the data suggest an alternative explanation when individual vulnerability or resistance to stress are analyzed as a result of the combination of the anxiety and exploration behavioral traits. While all of the groups showed similar reductions in body weight with repeated exposure to stress, at the behavioral level, the animals with the 'low anxiety and low exploration' profile were less vulnerable to alterations in sociability measures following different lengths of stress exposure. In contrast, the other three personality profiles showed a progressive reduction in social motivation, which was already apparent after two weeks of stress. The 'low anxiety and low exploration' group, in addition to the other low anxiety group (the 'low anxiety and high exploration' profile), also showed higher resilience to increases in floating time in the forced swim test. While the two high anxiety groups showed enhanced floating after only 2 weeks of stress, changes in this parameter were only significant for the animals with the low anxiety profiles after 4 weeks of stress. Thus, at the behavioral level, the high anxiety trait seems to increase the animal's likelihood to develop depression-like behaviors, which is consistent with the literature from both animals and humans (Kendler et al., 2006; see Section 1). Additionally, the low anxiety trait in combination with the low exploration trait provided resilience against developing stress-induced depression-like behaviors. Importantly, behavioral responses in tests for anxiety (Stiller et al., 2011), exploration (Minor et al., 1994; Walker et al., 2008) and stress coping (Drugan et al., 1989) in rats had been previously shown to predict the development of certain stress-induced depressive-like and/or conditioned fear behaviors. In addition, individual differences in novelty reactivity - generally termed "novelty-seeking" trait - in rats were found to be associated with the emergence of anxiety- and depression-like behaviors following repeated exposure to social defeat (Duclot et al., 2011). Specifically, animals that display a low reactivity to novelty were found to have a higher vulnerability to develop those psychopathological behaviors than rats displaying a high locomotor reactivity. In this connection, individual differences in novelty-seeking behavior in rats were also shown to predict differential responses to the antidepressant desipramine in the forced swim test (Jama et al., 2008).

The impact of stress on these behavioral profiles was further examined at the physiological and neurobiological levels. Interestingly, the 'high anxiety and low exploration' profile (one of the two highly vulnerable groups at the behavioral level) was the only profile that showed enhanced plasma corticosterone levels and pERK1/2 expression in 
amygdala and hippocampus (in virtually all of the other cases, these levels were reduced) following acute stress exposure. It should be noted that our corticosterone measurement $15 \mathrm{~min}$ after acute stress exposure is a readout of the maximum capacity of the adrenal gland to release this hormone after exposure to CUS, which is normally increased by chronic stress (Marquez et al., 2004). This is consistent with the fact that the higher corticosterone response was found in the most vulnerable group from a behavioral perspective. Our findings, therefore, seem to be in line with recent work suggesting that PERK activity in the amygdala is an inverse regulator of the animal's habituation to stress (Grissom and Bhatnagar, 2011). Following 2 weeks of exposure to restraint stress [a higher intensity stress protocol than CUS in terms of circuitry impact (Vyas et al., 2002)], stressinduced depression-like behaviors were correlated with an increase in pERK1/2 in the hippocampus (Bravo et al., 2009). The highest levels of $\mathrm{pERK}$ activation in the amygdala were found when the animals were submitted to uncontrollable stress, whereas it was reduced when they learn to control the stressor and were less anxious (Ilin and Richter-Levin, 2009). Pharmacological inhibition of pERK activation was shown to reverse depression-like behaviors in a CRF-2-deficient mouse model of depression (Todorovic et al., 2009). Further evidence links sustained increases in pERK1/2 with increased anxiety, as shown by intra-amygdala infusions of d-cycloserine (Wu et al., 2008). Additionally, it is interesting to note that individual differences in coping with predator stress - as manifested by post-stress anxiety responses in the elevated plus maze - were previously found in the literature to correlate with differences in the structure of the dendritic trees in the basolateral amygdala; thus, maladapted (extremely anxious) rats showed larger dendrites than welladapted (less anxious) animals (Mitra et al., 2009). Corticosterone treatment was found to mimic dendritic hypertrophy in the BLA and the enhanced anxiety observed after chronic stress (Mitra and Sapolsky, 2008). Differences in corticosterone responses and in neural activity in the amygdala were also previously found to be associated with coping responses to stress, with active coping leading to lower activation on those parameters (Walker et al., 2009).

In summary, our findings indicate that the high behavioral vulnerability of the 'high anxiety and low exploration' group was accompanied by this group's vulnerability to physiological and neurobiological indexes of anxiety and maladaptation to repeated stress. These characteristics resemble a behavioral inhibition profile that has been linked to significant differences in stress physiology and is associated with acute and basal glucocorticoid overproduction (Cavigelli et al., 2007). These findings suggest that the interaction between high anxiety and low exploration traits represent a higher risk to the effects of stress in congruence with the human traits of neuroticism and extraversion, which have been suggested to be predictors of an individual's vulnerability or resilience to develop depression (Lara and Akiskal, 2006).

In contrast, the other three experimental groups (both low anxiety groups and the 'high anxiety and high exploration' group) showed, overall, a mild reduction of $\mathrm{pERK} 1 / 2$ responses after 2 weeks of stress that was followed by a subsequent recovery to control levels with further stress exposure. This finding is consistent with experimental evidence that suggests that a reduction in pERK1/2 activity might indicate adaptation to repeated stress. Whereas acute exposure to stressors has been shown to lead to enhanced pERK activation (Ilin and Richter-Levin, 2009; GutièrrezMecinas et al., 2011), repeated exposure to stress for 5 consecutive days reduced pERK expression in the BLA, which was prevented by a $\beta$-adrenergic receptor blockade (Grissom and Bhatnagar, 2011). In animals that are well-trained in a cued avoidance response, exposure to the cue was found to reduce pERK levels in the amygdala (Botreau and GisquetVerrier, 2006). Importantly, the inhibition of PERK in the BLA mimicked the neuroendocrine and behavioral habituation induced by repeated exposure to stress (Grissom and Bhatnagar, 2011).

Altogether, our findings confirm the increased vulnerability of animals with high anxiety to certain behavioral alterations associated with depression (e.g., learned helplessness in the forced swim test) when progressively exposed to stress. Additionally, our findings suggest that the behavioral profile that combines 'low anxiety and low exploration' might be resistant to the development of behavioral alterations related to social behaviors. Among the highly anxious animals, the animals that showed a behavioral profile characterized by behavioral inhibition (i.e., the 'high anxiety and low exploration' group) displayed a particularly vulnerable pattern of physiological (higher corticosterone levels than the other groups when exposed to acute stress following 2 weeks of CUS) and neurobiological responses (increased pERK1/2 activation in the amygdala and hippocampus with progressive exposure to stress, as opposed to the other groups that showed an initial reaction followed by a return to control levels by the 4th week of CUS). Overall, the differential vulnerability observed among the different behavioral profiles was particularly evident after the sub-chronic exposure to stress (i.e., 2 weeks), whereas the animals' responses after further stress exposure (i.e., 4 weeks) tended to be similarly affected. Our findings suggest that individual vulnerability and/or resilience to the effects of stress might be particularly apparent during the initial or intermediate levels of stress exposure, and they highlight that the behavioral inhibition profile of an animal indicates whether it is likely to be susceptible to responding in a deleterious manner to stress. Thus, we provide a relevant model to further investigate key neurobiological mechanisms that underlie vulnerability and resilience to stress.

\section{Role of the funding sources}

The founding sources have contributed to the development of the research but do not have any interest in the results. They have not participated in the specific design of experiments and are not involved in the exploitation of the obtained data.

\section{Conflict of interest}

The authors declare not to have any conflict of interest.

\section{Acknowledgments}

We thank Coralie Siegmund, Mark Fajans, Laura Leon and Lorena Moreno for their excellent technical assistance. This work was supported by grants from the Swiss National Science 
Foundation (310000-120791 and 31003AB-135710, Sinergia CRSIK3-122691 and CRSIK0-122697, and the NCCR "The synaptic basis of mental diseases"), the FP7 Health program (MemStick) and intramural funding from the EPFL.

\section{Appendix A. Supplementary data}

Supplementary data associated with this article can be found, in the online version, at doi:10.1016/j.psyneuen. 2011.12.014.

\section{References}

Adler, L., Wedekind, D., Pilz, J., Weniger, G., Huether, G., 1997. Endocrine correlates of personality traits: a comparison between emotionally stable and emotionally labile healthy young men. Neuropsychobiology 35, 205-210.

Ball, S.A., 1995. The validity of an alternative five-factor measure of personality in cocaine abusers. Psychol. Assess. 7, 148-154.

Ball, S.A., Schottenfeld, R.S., 1997. A five-factor model of personality and addiction, psychiatric, and AIDS risk severity in pregnant and postpartum cocaine misusers. Subst. Use Misuse 32, 25-41.

Ballenger, J.C., Post, R.M., Jimerson, D.C., Lake, C.R., Murphy, D.L., Zuckerman, M., Cronin, C., 1983. Biochemical correlates of personality traits in normals: an exploratory study. Pers. Indiv. Differ. 4, 615-625.

Bisaz, R., Schachner, M., Sandi, C., 2011. Causal evidence for the involvement of the neural cell adhesion molecule, NCAM, in chronic stress-induced cognitive impairments. Hippocampus 21 (1), 56-71.

Botreau, F., Gisquet-Verrier, P., 2006. Memory reactivation, dissociated from behavioural expression, decreases ERK phosphorylation in the rat prefrontal cortex and amygdala. Behav. Brain Res. 169 (1), 176-180.

Bravo, J.A., Díaz-Veliz, G., Mora, S., Ulloa, J.L., Berthoud, V.M., Morales, P., Arancibia, S., Fiedler, J.L., 2009. Desipramine prevents stress-induced changes in depressive-like behavior and hippocampal markers of neuroprotection. Behav. Pharmacol. 20 (3), 273-285.

Castro, J.E., Varea, E., Márquez, C., Cordero, M.I., Poirier, G., Sandi, C., 2010. Role of the amygdala in antidepressant effects on hippocampal cell proliferation and survival and on depressionlike behavior in the rat. PLoS One 5 (1), e8618.

Cavigelli, S.A., Stine, M.M., Kovacsics, C., Jefferson, A., Diep, M.N., Barrett, C.E., 2007. Behavioral inhibition and glucocorticoid dynamics in a rodent model. Physiol. Behav. 92 (5), 897-905.

Conrad, C.D., LeDoux, J.E., Magariños, A.M., McEwen, B.S., 1999. Repeated restraint stress facilitates fear conditioning independently of causing hippocampal CA3 dendritic atrophy. Behav. Neurosci. 113 (5), 902-913.

Cordero, M.I., Rodríguez, J.J., Davies, H.A., Peddie, C.J., Sandi, C., Stewart, M.G., 2005. Chronic restraint stress down-regulates amygdaloid expression of polysialylated neural cell adhesion molecule. Neuroscience 133 (4), 903-910.

Crawley, J., Goodwin, F.K., 1980. Preliminary report of a simple animal behavior model for the anxiolytic effects of benzodiazepines. Pharmacol. Biochem. Behav. 13 (2), 167-170.

de Kloet, C.S., Vermetten, E., Heijnen, C.J., Geuze, E., Lentjes, E.G., Westenberg, H.G., 2007. Enhanced cortisol suppression in response to dexamethasone administration in traumatized veterans with and without post-traumatic stress disorder. Psychoneuroendocrinology 32 (3), 215-226.

de Kloet, E.R., Joëls, M., Holsboer, F., 2005. Stress and the brain: from adaptation to disease. Nat. Rev. Neurosci. 6, 463-475.

de Rijk, R., de Kloet, E.R., 2005. Corticosteroid receptor genetic polymorphisms and stress responsivity. Endocrine 28 (3), 263-270.
Doremus, T.L., Varlinskaya, E.I., Spear, L.P., 2006. Factor analysis of elevated plus-maze behavior in adolescent and adult rats. Pharmacol. Biochem. Behav. 83, 570-577.

Drevets, W.C., Price, J.L., Simpson, J.R., Todd, R.D., Reich, T., Vannier, M., 1997. Subgenual prefrontal cortex abnormalities in mood disorders. Nature 386 (6627), 824-827.

Drugan, R.C., Skolnick, P., Paul, S.M., Crawley, J.N., 1989. A pretest procedure reliably predicts performance in two animal models of inescapable stress. Pharmacol. Biochem. Behav. 33 (3), 649-654.

Duclot, F., Hollis, F., Darcy, M.J., Kabbaj, M., 2011. Individual differences in novelty-seeking behavior in rats as a model for psychosocial stress-related mood disorders. Physiol. Behav. 104 (2), 296-305.

Feder, A., Nestler, E.J., Charney, D.S., 2009. Psychobiology and molecular genetics of resilience. Nat. Rev. Neurosci. 10 (6), 446-457.

Fendt, M., Endres, T., 2008. 2,3,5-Trimethyl-3-thiazoline (TMT), a component of fox odor - just repugnant or really fear-inducing? Neurosci. Biobehav. Rev. 32 (7), 1259-1266.

Fuss, J., Ben Abdallah, N.M., Vogt, M.A., Touma, C., Pacifici, P.G., Palme, R., 2010. Voluntary exercise induces anxiety-like behavior in adult C57BL/6J mice correlating with hippocampal neurogenesis. Hippocampus 20 (3), 364-376.

Grissom, N.M., Bhatnagar, S., 2011. The basolateral amygdala regulates adaptation to stress via $\beta$-adrenergic receptor-mediated reductions in phosphorylated extracellular signal-regulated kinase. Neuroscience 31 (178), 108-122.

Gunnar, M.R., Tout, K., De Haan, M., Pierce, S., Stansbury, K., 1997. Temperament, social competence, and adrenocortical activity in preschoolers. Dev. Psychobiol. 31, 65-85.

Gutièrrez-Mecinas, M., Trollope, A.F., Collins, A., Morfett, H., Hesketh, S.A., Kersanté, F., Reul, J.M., 2011. Long-lasting behavioral responses to stress involve a direct interaction of glucocorticoid receptors with ERK1/2-MSK1-Elk-1 signaling. Proc. Natl. Acad. Sci. U. S. A. 108 (33), 13806-13811.

Hennig, J., 2004. Personality, serotonin, and noradrenaline. In: Stelmack, M. (Ed.), On the Psychobiology of Personality, vol. 20. Elsevier B.V., Amsterdam, pp. 379-408.

Herrero, A.I., Sandi, C., Venero, C., 2006. Individual differences in anxiety trait are related to spatial learning abilities and hippocampal expression of mineralocorticoid receptors. Neurobiol. Learn. Mem. 86 (2), 150-159.

Holmes, A., Wellman, C.L., 2009. Stress-induced prefrontal reorganization and executive dysfunction in rodents. Neurosci. Biobehav. Rev. 33 (6), 773-783.

Ilin, Y., Richter-Levin, G., 2009. Enriched environment experience overcomes learning deficits and depressive-like behavior induced by juvenile stress. PLoS One 4 (1), e4329.

Jama, A., Cecchi, M., Calvo, N., Watson, S.J., Akil, H., 2008. Interindividual differences in novelty-seeking behavior in rats predict differential responses to desipramine in the forced swim test. Psychopharmacology (Berl) 198 (3), 333-340.

Kendler, K.S., Gatz, M., Gardner, C.O., Pedersen, N.L., 2006. Personality and major depression: a Swedish longitudinal, population-based twin study. Arch. Gen. Psychiatry 63 (10), 1113-1120.

Lara, D.R., Akiskal, H.S., 2006. Toward an integrative model of the spectrum of mood, behavioral and personality disorders based on fear and anger traits: II. Implications for neurobiology, genetics and psychopharmacological treatment. J. Affect. Disord. 94 (13), 89-103.

Larsen, M.H., Mikkelsen, J.D., Hay-Schmidt, A., Sandi, C., 2010. Regulation of brain-derived neurotrophic factor (BDNF) in the chronic unpredictable stress rat model and the effects of chronic antidepressant treatment. J. Psychiatr. Res. 44 (13), 808-816.

Lindfors, P., Lundberg, U., 2002. Is low cortisol release an indicator of positive health. Stress Health 18, 153-160.

Lupien, S.J., de Leon, M., de Santi, S., Convit, A., Tarshish, C., Nair, N.P., et al., 1998. Cortisol levels during human aging predict 
hippocampal atrophy and memory deficits. Nat. Neurosci. 1 (1), 69-73.

Magariños, A.M., McEwen, B.S., 1995. Stress-induced atrophy of apical dendrites of hippocampal CA3C neurons: comparison of stressors. Neuroscience 69 (1), 83-88.

Marquez, C., Nadal, R., Armario, A., 2004. The hypothalamic-pituitary-adrenal and glucose responses to daily repeated immobilisation stress in rats: individual differences. Neuroscience 123 (3), 601-612.

Mazur, A., 1995. Biosocial models of deviant behavior among male army veterans. Biol. Psychol. 41, 271-293.

Mazzucchelli, C., Brambilla, R., 2000. Ras-related and MAPK signalling in neuronal plasticity and memory formation. Cell. Mol. Life Sci. 57 (4), 604-611.

McEwen, B.S., 2000. Effects of adverse experiences for brain structure and function. Biol. Psychiatry 48 (8), 721-731.

McEwen, B.S., 2003. Early life influences on life-long patterns of behavior and health. Ment. Retard. Dev. Disabil. Res. Rev. 9 (3), 149-154.

McEwen, B.S., 2004. Protection and damage from acute and chronic stress: allostasis and allostatic overload and relevance to the pathophysiology of psychiatric disorders. Ann. N. Y. Acad. Sci. 1032, 1-7.

Minor, T.R., Dess, N.K., Ben-David, E., Chang, W.-C., 1994. Individual differences in vulnerability to inescapable shock in rats. J. Exp. Psychol. Anim. Behav. Process. 20 (4), 402-412.

Mitra, R., Ferguson, D., Sapolsky, R.M., 2009. SK2 potassium channel overexpression in basolateral amygdala reduces anxiety, stressinduced corticosterone secretion and dendritic arborization. Mol. Psychiatry 14 (9), 847-855 827.

Mitra, R., Jadhav, S., McEwen, B.S., Vyas, A., Chattarji, S., 2005. Stress duration modulates the spatiotemporal patterns of spine formation in the basolateral amygdala. Proc. Natl. Acad. Sci. U. S. A. 102 (26), 9371-9376.

Mitra, R., Sapolsky, R.M., 2008. Acute corticosterone treatment is sufficient to induce anxiety and amygdaloid dendritic hypertrophy. Proc. Natl. Acad. Sci. U. S. A. 105 (14), 5573-5578.

New, A.S., Fan, J., Murrough, J.W., Liu, X., Liebman, R.E., Guise, K.G., Tang, C.Y., Charney, D.S., 2009. A functional magnetic resonance imaging study of deliberate emotion regulation in resilience and posttraumatic stress disorder. Biol. Psychiatry 66 (7), 656-664.

Oitzl, M.S., Champagne, D.L., van der Veen, R., de Kloet, E.R., 2010. Brain development under stress: hypotheses of glucocorticoid actions revisited. Neurosci. Biobehav. Rev. 34 (6), 853-856.

Oitzl, M.S., Workel, J.O., Fluttert, M., Frosch, F., de Kloet, E.R., 2000. Maternal deprivation affects behaviour from youth to senescence: amplification of individual differences in spatial learning and memory in senescent Brown Norway rats. Eur. J. Neurosci. 12 (10), 3771-3780.

Pham, K., Nacher, J., Hof, P.R., McEwen, B.S., 2003. Repeated restraint stress suppresses neurogenesis and induces biphasic PSA-NCAM expression in the adult rat dentate gyrus. Eur. J. Neurosci. 17 (4), 879-886.

Piazza, P.V., Deminiere, J.M., Le Moal, M., Simon, H., 1989. Factors that predict individual vulnerability to amphetamine self-administration. Science 245 (4925), 1511-1513.

Pickering, A.D., 2004. The neuropsychology of impulsive antisocial sensation seeking personality traits: from dopamine to hippocampal function? In: Stelmack, M. (Ed.), On the Psychobiology of Personality, vol. 23. Elsevier B.V., Amsterdam, pp. 459-476.

Porsolt, R.D., Le Pichon, M., Jalfre, M., 1977. Depression: a new animal model sensitive to antidepressant treatments. Nature 266 (5604), 730-732.

Qi, X., Lin, W., Li, J., Pan, Y., Wang, W., 2006. The depressive-like behaviors are correlated with decreased phosphorylation of mitogen-activated protein kinases in rat brain following chronic forced swim stress. Behav. Brain Res. 175, 233-240.
Rajkowska, G., 2000. Histopathology of the prefrontal cortex in major depression: what does it tell us about dysfunctional monoaminergic circuits? Prog. Brain Res. 126, 397-412.

Refojo, D., Echenique, C., Muller, M.B., Reul, J.M., Deussing, J.M., Wurst, W., Sillaber, I., Paez-Pereda, M., Holsboer, F., Arzt, E., 2005. Corticotropin-releasing hormone activates ERK1/2 MAPK in specific brain areas. Proc. Natl. Acad. Sci. U. S. A. 102, 61836188.

Renthal, W., Maze, I., Krishnan, V., Covington 3rd, H.E., Xiao, G., Kumar, A., et al., 2007. Histone deacetylase 5 epigenetically controls behavioral adaptations to chronic emotional stimuli. Neuron 56 (3), 517-529.

Reul, J.M., Chandramohan, Y., 2007. Epigenetic mechanisms in stress-related memory formation. Psychoneuroendocrinology 32 (Suppl. 1), S21-S25.

Revest, J.M., Dupret, D., Koehl, M., Funk-Reiter, C., Grosjean, N., Piazza, P.V., et al., 2009. Adult hippocampal neurogenesis is involved in anxiety-related behaviors. Mol. Psychiatry 14, 959967.

Roozendaal, B., McEwen, B.S., Chattarji, S., 2009. Stress, memory and the amygdala. Nat. Rev. Neurosci. 10, 423-433.

Rosenblitt, J.C., Soler, H., Johnson, S.E., Quadagno, D.M., 2001. Sensation seeking and hormones in men and women: exploring the link. Horm. Behav. 40, 396-402.

Rotllant, D., Márquez, C., Nadal, R., Armario, A., 2010. The brain pattern of c-fos induction by two doses of amphetamine suggests different brain processing pathways and minor contribution of behavioural traits. Neuroscience 168 (3), 691-705.

Rudolph, K.D., Hammen, C., 1999. Age and gender as determinants of stress exposure, generation, and reactions in youngsters: a transactional perspective. Child Dev. 70 (3), 660-677.

Salehi, B., Cordero, M.I., Sandi, C., 2010. Learning under stress: the inverted-U-shape function revisited. Learn. Mem. 17 (10), 522530.

Sandi, C., Cordero, M.I., Ugolini, A., Varea, E., Caberlotto, L., et al., 2008. Chronic stress-induced alterations in amygdala responsiveness and behavior-modulation by trait anxiety and corticotropinreleasing factor systems. Eur. J. Neurosci. 28, 1836-1848.

Sandi, C., Richter-Levin, G., 2009. From high anxiety trait to depression: a neurocognitive hypothesis. Trends Neurosci. 32, 312320.

Sheline, Y.I., Sanghavi, M., Mintun, M.A., Gado, M.H., 1999. Depression duration but not age predicts hippocampal volume loss in medically healthy women with recurrent major depression. J. Neurosci. 19 (12), 5034-5043.

Shen, C.P., Tsimberg, Y., Salvadore, C., Meller, E., 2004. Activation of Erk and JNK MAPK pathways by acute swim stress in rat brain regions. BMC Neurosci. 5, 36.

Silberstein, S., Vogl, A.M., Refojo, D., Senin, S.A., Wurst, W., Holsboer, F., Deussing, J.M., Arzt, E., 2009. Amygdaloid pERK1/2 in corticotropin-releasing hormone overexpressing mice under basal and acute stress conditions. Neuroscience 159, 610617.

Southwick, S.M., Vythilingam, M., Charney, D.S., 2005. The psychobiology of depression and resilience to stress: implications for prevention and treatment. Annu. Rev. Clin. Psychol. 1, 255-291.

Stam, R., 2007. PTSD and stress sensitisation: a tale of brain and body Part 2: animal models. Neurosci. Biobehav. Rev. 31 (4), 558-584.

Stiller, A.L., Drugan, R.C., Hazi, A., Kent, S.P., 2011. Stress resilience and vulnerability: the association with rearing conditions, endocrine function, immunology, and anxious behavior. Psychoneuroendocrinology 36 (9), 1383-1395.

Todorovic, C., Sherrin, T., Pitts, M., Hippel, C., Rayner, M., Spiess, J., 2009. Suppression of the MEK/ERK signaling pathway reverses depression-like behaviors of CRF2-deficient mice. Neuropsychopharmacology 34 (6), 1416-1426.

Tyrka, A.R., Wier, L.M., Price, L.H., Rikhye, K., Ross, N.S., Anderson, G.M., Wilkinson, C.W., Carpenter, L.L., 2008. Cortisol and ACTH 
responses to the Dex/CRH test: influence of temperament. Horm. Behav. 53 (4), 518-525.

Vyas, A., Jadhav, S., Chattarji, S., 2006. Prolonged behavioral stress enhances synaptic connectivity in the basolateral amygdala. Neuroscience 143 (2), 387-393.

Vyas, A., Mitra, R., ShankaranarayanaRao, B.S., Chattarji, S., 2002. Chronic stress induces contrasting patterns of dendritic remodeling in hippocampal and amygdaloid neurons. J. Neurosci. 22 (15), 6810-6818.

Vyas, A., Pillai, A.G., Chattarji, S., 2004. Recovery after chronic stress fails to reverse amygdaloid neuronal hypertrophy and enhanced anxiety-like behavior. Neuroscience 128 (4), 667-673.

Walker, F.R., Masters, L.M., Dielenberg, R.A., Day, T.A., 2009. Coping with defeat: acute glucocorticoid and forebrain responses to social defeat vary with defeat episode behaviour. Neuroscience 162 (2), 244-253.

Walker, F.R., Hinwood, M., Masters, L., Deilenberg, R.A., Day, T.A., 2008. Individual differences predict susceptibility to conditioned fear arising from psychosocial trauma. J. Psychiatr. Res. 42 (5), 371-383.
Wang, W., Du, W., Lieu, P., Lieu, J., Wang, Y., 2002. Five-factor measures in Chinese university students: effects of one-child policy? Psychiatry Res. 109, 37-44.

Wang, W., Du, W., Wang, Y., Livesley, W.J., Jang, K.L., 2004. The relationship between the Zuckerman-Kuhlman Personality Questionnaire and traits delineating personality pathology. Pers. Indiv. Differ. 36, 155-162.

Willner, P. , 2005. Chronic mild stress (CMS) revisited: consistency and behavioural-neurobiological concordance in the effects of CMS. Neuropsychobiology 52 (2), 90-110.

Willner, P., 1997. Validity, reliability and utility of the chronic mild stress model of depression: a 10-year review and evaluation. Psychopharmacology (Berl) 134, 319-329.

Wu, S.L., Hsu, L.S., Tu, W.T., Wang, W.F., Huang, Y.T., Pawlak, C.R., Ho, Y.J., 2008. Effects of D-cycloserine on the behavior and ERK activity in the amygdala: role of individual anxiety levels. Behav. Brain Res. 187, 246-253.

Yang, R.J., Mozhui, K., Karlsson, R.M., Cameron, H.A., Williams, R.W., et al., 2008. Variation in mouse basolateral amygdala volume is associated with differences in stress reactivity and fear learning. Neuropsychopharmacology 33, 2595-2604. 